\title{
Breaking out of the Box: Wearable Technology Applications for Detecting the Spread of COVID-19
}

\author{
Mostafa Al-Emran $^{1}$ (D) . Jesse M. Ehrenfeld ${ }^{2}$
}

Received: 6 December 2020 / Accepted: 7 December 2020 / Published online: 11 January 2021

(C) The Author(s), under exclusive licence to Springer Science+Business Media, LLC part of Springer Nature 2021

The novel coronavirus, COVID-19, caused by SARSCoV-2 first detected in Wuhan, China, in December 2019, has become a global pandemic. With the exponential increase of patients affected by COVID-19 and the absence of an effective therapy or vaccine, COVID-19 still represents a global health challenge [1]. Traditional public health tools (e.g., testing, contact tracing, and social distancing) still remain the most efficient tools to combat the pandemic and slow its spread. When an individual is being quarantined, it is essential to monitor his/ her symptoms and temperature. While this approach mainly depends on the individual's self-discipline, it can't be fully controlled and is subject to the individuals' capacity and compliance. However, the emergence of smart wearable technologies makes it feasible to monitor individuals' physiological parameters using wearable sensors at scale in ways that could shift the trajectory of the pandemic.

Wearable technologies refer to the smart electronic devices that are worn on the body to evaluate and transfer various types of data, such as signals related to human bodies and their physical activities [2]. While wearable technologies have been widely cited as an effective approach in healthcare, the emergence of COVID-19 presents an opportunity to use these wearables to contain the spread of the disease. Those

This article is part of the Topical Collection on Mobile \& Wireless Health

Mostafa Al-Emran

mustafa.n.alemran@gmail.com

Jesse M. Ehrenfeld

jehrenfeld@mcw.edu

1 Faculty of Engineering \& IT, The British University in Dubai, Dubai, UAE

2 Advancing a Healthier Wisconsin Endowment, Medical College of Wisconsin, Milwaukee, WI, USA wearables have the potential to provide real-time remote monitoring and symptoms prediction based on blood oxygen saturation ( $\mathrm{SpO} 2)$ level, body temperature, blood pressure, lung sound, and heart rate. Relying on their effective sensors, those wearables have been used to alert individuals of a potential COVID-19 infection prior to the appearance of the severe symptoms [3]. Those wearables were also used to monitor the multidimensional physiological parameters for early detecting the progression of COVID-19 in patients [4].

While there are other applications of wearable technologies, they have not yet been used during the pandemic. Given the tiny size of COVID-19 cells, approximately $0.12 \mu \mathrm{m}$ in diameter, a microscope is required to visualize the particles. Smart wearable glasses could however be used to detect the spread of COVID-19, whether in the air or human bodies by equipping glasses with sensors that have the same characteristics as a microscope to detect the movement of COVID-19 cells. Those wearables might also be used to reduce the infection rate by providing early screening for those vulnerable or exposed to infection. Due to the availability of real-time data, wearable technologies might also be used to hinder the transmission rate of COVID-19 among patients, physicians, and other healthcare workers. Those wearables might also be applied to deliver telehealth and telemedicine services through their biosensors.

It is important to recognize that COVID-19 will not be the last pandemic to challenge the world's population and our healthcare systems. The evolution of artificial intelligence applications has played a crucial role in predicting, identifying, and preventing the risk of COVID-19, and we must also consider its role in preventing future pandemics. The availability of the data generated through wearable technologies could be effectively used to predict the infection of COVID19 through machine learning algorithms. The effect of wearable technologies on COVID-19 is real, and their potential to detect, identify, and prevent its spread is applicable only when more research efforts are put forward. The current global pandemic has clearly created an opportunity for many 
developers to think out of the box by developing wearable devices that better serve the healthcare sector, now and in the future.

\section{References}

1. Whitelaw, S., Mamas, M. A., Topol, E., Van Spall, H. G. C., Applications of digital technology in COVID-19 pandemic planning and response. Lancet Dig. Health. 2020, https://doi.org/10.1016/ S2589-7500(20)30142-4.

2. Islam, M. M., Mahmud, S., Muhammad, L. J., Islam, M. R., Nooruddin, S., Ayon, S. I., Wearable Technology to Assist the
Patients Infected with Novel Coronavirus (COVID-19). SN Comput. Sci. 1(6):1-9, 2020.

3. Seshadri, D. R., et al., Wearable Sensors for COVID-19: A Call to Action to Harness Our Digital Infrastructure for Remote Patient Monitoring and Virtual Assessments. Front. Digit. Heal. 2:1-11, 2020, https://doi.org/10.3389/fdgth.2020.00008.

4. Wong, C. K. et al., Artificial intelligence mobile health platform for early detection of COVID-19 in quarantine subjects using a wearable biosensor: Protocol for a randomised controlled trial. BMJ Open 10(7):1-5, 2020, https://doi.org/10.1136/bmjopen-2020-038555.

Publisher's Note Springer Nature remains neutral with regard to jurisdictional claims in published maps and institutional affiliations. 